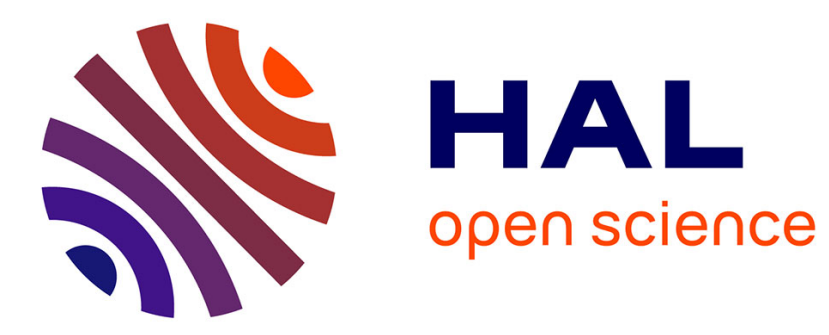

\title{
An experimental study of particle sedimentation using ultrasonic speckle velocimetry
}

Maxime Chinaud, Thomas Delaunay, Philippe Tordjeman

\section{To cite this version:}

Maxime Chinaud, Thomas Delaunay, Philippe Tordjeman. An experimental study of particle sedimentation using ultrasonic speckle velocimetry. Measurement Science and Technology, 2010, vol. 21, 10.1088/0957-0233/21/5/055402 . hal-00909563

\section{HAL Id: hal-00909563 \\ https://hal.science/hal-00909563}

Submitted on 26 Nov 2013

HAL is a multi-disciplinary open access archive for the deposit and dissemination of scientific research documents, whether they are published or not. The documents may come from teaching and research institutions in France or abroad, or from public or private research centers.
L'archive ouverte pluridisciplinaire HAL, est destinée au dépôt et à la diffusion de documents scientifiques de niveau recherche, publiés ou non, émanant des établissements d'enseignement et de recherche français ou étrangers, des laboratoires publics ou privés. 


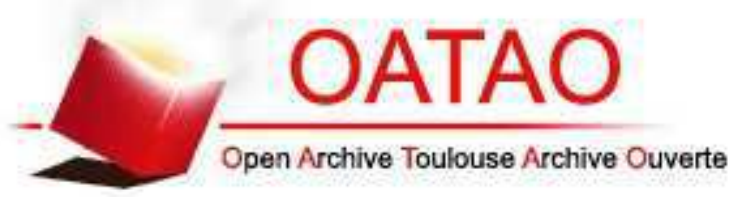

\section{Open Archive TOULOUSE Archive Ouverte (OATAO)}

OATAO is an open access repository that collects the work of Toulouse researchers and makes it freely available over the web where possible.

This is an author-deposited version published in : http://oatao.univ-toulouse.fr/ Eprints ID : 10271

To link to this article : doi:10.1088/0957-0233/21/5/055402

URL : http://dx.doi.org/10.1088/0957-0233/21/5/055402

To cite this version : Chinaud, Maxime and Delaunay, Thomas and Tordjeman, Philippe An experimental study of particle sedimentation using ultrasonic speckle velocimetry. (2010) Measurement Science and Technology, vol. $21\left(\mathrm{n}^{\circ}\right.$ 5). ISSN 0957-0233

Any correspondance concerning this service should be sent to the repository administrator: staff-oatao@ listes-diff.inp-toulouse.fr 


\title{
An experimental study of particle sedimentation using ultrasonic speckle velocimetry
}

\author{
M Chinaud $^{1}$, T Delaunay ${ }^{1}$ and Ph Tordjeman ${ }^{2,3}$ \\ ${ }^{1}$ Laboratoire IES, Groupe Micro et RhéoAcoustique, UMR CNRS 5214, Université Montpellier 2, \\ CC 082, Pl. E Bataillon, 34095 Montpellier Cedex 05, France \\ ${ }^{2}$ Université de Toulouse, INPT-CNRS, Institut de Mécanique des Fluides (IMFT), 1 Allée du Professeur \\ Camille Soula, 31400 Toulouse, France \\ E-mail: philippe.tordjeman@imft.fr
}

\begin{abstract}
Ultrasonic speckle velocimetry (USV) is a non-invasive technique that allows the measurement of fluid velocity in flow and also that of powders under sedimentation. To improve the USV method, we studied the sedimentation of polymethyl methacrylate and silica particles in water. Then, we built a sedimentation cell and characterized the diameter distribution of the particles. Subsequently, we carried out a specific study to optimize the USV procedure, the signal processing and data analysis. Space and temporal resolution and USV dynamics are also discussed with regard to the optimization conditions. We found that USV is a useful technique to measure velocities between $10^{-5}$ and $1 \mathrm{~m} \mathrm{~s}^{-1}$, using appropriate ultrasonic transducers. The space resolution is fixed by the length and the percentage of overlapping of the analyzed speckle windows and varies between 48 and $536 \mu \mathrm{m}$ for the different studied particle families. Furthermore, we found that a $0.1 \mathrm{~ns}$ temporal resolution could be obtained after a zero padding signal processing. In the context of our sedimentation experiments, we showed that the velocities measured by USV are in close agreement with those measured by particle image velocimetry and theory.
\end{abstract}

Keywords: ultrasonic speckle velocimetry, particle sedimentation, signal processing, data analysis

\section{Introduction}

The measurement of velocity fields for complex fluids is a challenge in many applications of fluids research, from the flow imagery in non-transparent geometries to the understanding of instabilities and turbulence. Ultrasonic techniques are noninvasive quantitative techniques and highly suited to the study of complex fluids due to their capacity to characterize opaque fluids compared to optical techniques such as particle image velocimetry (PIV) [1, 2]. There are two main ultrasonic methods: Doppler echo ultrasound techniques and ultrasonic speckle techniques. The first technique is widely used in

\footnotetext{
3 Author to whom any correspondence should be addressed.
}

hydrodynamics, in particular, in clinical applications [3, 4]: the Doppler shift of ultrasound echoes reflected by blood cells allows measuring the flow velocity component along the ultrasound beam [5]. Sweeping the beam by a mechanical or an electronic system provides a two-dimensional map of the velocity component. Recent studies [6] propose an alternative method using the analysis of ultrasound echo images with a conventional optical PIV technique. The latter methods produce interesting results but are hindered by complex electronic systems.

The second principle of acoustic velocimetry techniques is based on the speckle dynamics, characterizing the ultrasonic field, which is scattered by moving contrast agents. The 
contrast agents are dispersed throughout a flowing fluid, and are exposed to a pulsed ultrasonic wave. Ultrasonic speckle velocimetry (USV) is a recently developed technique [7] and does not require the development of a new transducer. Recently [8-10], USV has been successfully applied to measure the multicomponent complex fluid velocity and characterize flowing instabilities such as the shear banding of opaque fluids in a rheological device. Despite its interesting applications, USV is not widely used for the measurement of flow velocities due to a lack of the signal processing description needed to improve the low signal-to-noise ratio of the ultrasonic backscattered response [11]. However, USV can be considered a high-performance method compared to Doppler methods, considering recent recording systems such as the digital oscilloscope associated with efficient signal processing. Through the study of particle sedimentation in a Newtonian fluid, this paper reports experimental procedures to optimize the USV measurements. Signal processing conditions and data analysis are carried out and discussed in terms of accuracy, dynamics and resolution.

\section{Methods}

\subsection{USV principles}

USV is a process whereby an ultrasonic pulse is backscattered by seeded particles in flowing fluids [7, 10]. Hence, if one considers an acoustic pulse, the incident pressure field can be written in terms of its amplitude $A(\vec{r})$ and its phase $\Phi(\vec{r}, t)$ :

$$
P(\vec{r}, t)=A(\vec{r}) \exp (\mathrm{i} \Phi(\vec{r}, t)) .
$$

The speckle intensity results from coherent mixing at the detector of all the acoustic waves backscattered by the seeded particles. In the context of the Huygens-Fresnel approximation, the temporal mean power measured by the piezoelectric transducer follows the well-known scaling relation:

$$
\left\langle P(\vec{r}) P^{*}(\vec{r})\right\rangle \approx R\left|\int \frac{P_{i}(\vec{r})}{r} \exp (\overrightarrow{\mathrm{i} k} \cdot \vec{r}) \mathrm{d} r\right|^{2},
$$

where $R$ is a reflection factor characteristic of the fluidparticles system and $\vec{k}$ is the ultrasonic wave vector $[12,13]$. Considering the ultrasonic transducer at the position $\vec{r}_{0}$, the time $t$ relative to the speckle signal at a distance $\vec{r}$ is given by $t=2 \frac{\left\|\vec{r}-\vec{r}_{0}\right\|}{c}$, with $c$ being the speed of sound in the fluid, and the time origin is defined here by the pulse emission. Note that the speckle signal $S(\vec{r})$ takes into account the attenuation with the distance and the transducer directivity:

$$
S(\vec{r})=\alpha(\vec{r})\left\langle P(\vec{r}) P^{*}(\vec{r})\right\rangle,
$$

where $\alpha(\vec{r})$ is the apodization factor that takes into account the transducer aperture. One can note that $S(\vec{r})$ can be obtained only with a particle density of ultrasound contrast agents varying between $10^{11}$ and $10^{12} \mathrm{~m}^{-3}$ [7]. Hence, the fluid velocity $\vec{v}(\vec{r})$ can be determined by the cross-correlation of two successive speckles separated by a pulse repetition time $t_{\mathrm{PRF}}$ according to (figure $1(a)$ )

$$
C(\vec{r})=\sum_{r-\Delta r}^{r+\Delta r} S(\vec{r}, 0) S\left(\vec{r}, t_{\mathrm{PRF}}\right),
$$
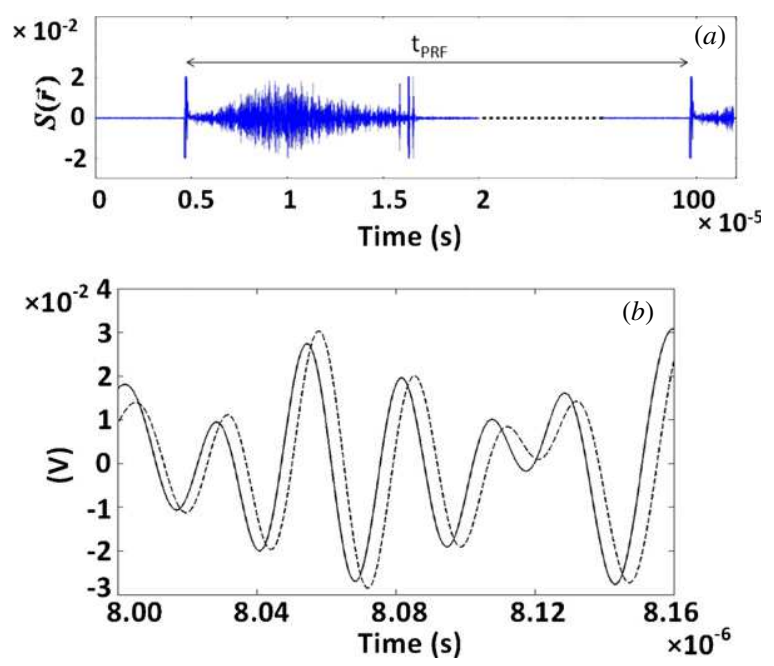

Figure 1. (a) Speckle signal recorded between two successive acoustical pulses. $t_{\mathrm{PRF}}$ is the time between the two pulses. (b) Zoom of two speckle signals at $t$ (solid line) and $t+t_{\mathrm{PRF}}$ (dashed line).

where $t_{\mathrm{PRF}}$ is a tunable parameter controlled by the pulserreceiver unit. Figure $1(a)$ presents the backscattered signal $S(\vec{r})$ between two successive pulse echoes and figure $1(b)$ shows the correlation between two successive speckle signals. $C(\vec{r})$ is calculated for a speckle window $\Delta r=n \lambda$, where $\lambda$ is the acoustic wavelength and $n$ is an integer typically varying between 2 and 20. The displacement of the scatterers at $\vec{r}$ is given by the time value $\delta t$ that maximizes $C(\vec{r})$. Finally, by assuming that the seeded particles behave as Lagrangian tracers, the fluid velocity along the ultrasonic beam axis is given by

$$
\vec{v}(\vec{r})=\frac{1}{2} c \frac{\delta t}{t_{\mathrm{PRF}}} .
$$

The flow velocity vector is obtained by the projection of the velocity vector along the acoustic axis.

\subsection{Particle sedimentation velocimetry}

In order to operate USV, we studied the sedimentation of particles in a Newtonian fluid. We developed a specific sedimentation setup which allows the USV measurements: the studied fluid is contained in a rectangular Plexiglas cell $\left(2.4 \times 3.6 \times 5 \mathrm{~cm}^{3}\right)$ fixed to an aluminium base. The acoustic transducer is placed in the base and the acoustic axis characteristic of the direction of the wave vector coincides with the sedimentation axis (figure 2). Waterproofness is obtained with a compressed toric seal. Two commercial ultrasonic linear transducers (Olympus V312-SM), with 2.25 and $25 \mathrm{MHz}$ frequencies, are used: their standard dimensions are $9.5 \mathrm{~mm}$ diameter, $32 \mathrm{~mm}$ length and $6 \mathrm{~mm}$ diameter of the element size. The transducers are controlled by a pulserreceiver generator Olympus Panamétrics $200 \mathrm{MHz}$ connected to a digital oscilloscope Lecroy, Wave Runner MXi6400, $10 \mathrm{GHz}$. Different speckle signals are recorded with the oscilloscope and triggered at a pulse repetition frequency, $\mathrm{PRF}=\left(t_{\mathrm{PRF}}\right)^{-1}$, tunable from 0 to $20 \mathrm{kHz}$. Data are subsequently forwarded to a computer and analysed by a Matlab ${ }^{\circledR}$ program. 


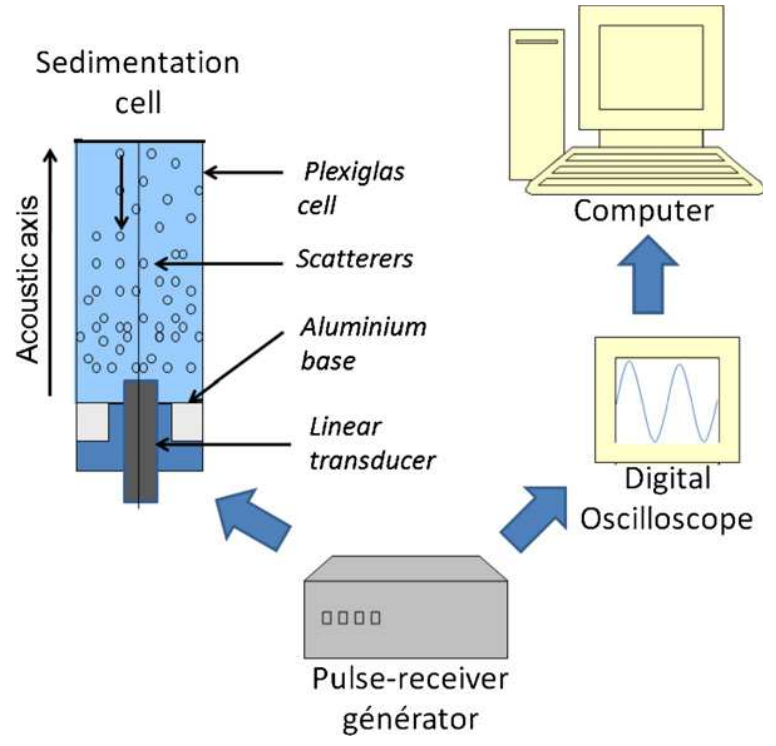

Figure 2. Scheme of the sedimentation device.

During the experimental procedure, particles are injected into water at $25{ }^{\circ} \mathrm{C}$ using a funnel at the top of the cell. This method allows distributing the particle population all around the cell and it also avoids cluster formation.

In this paper, we studied the sedimentation of three different particle populations.

- Polymethyl methacrylate (PMMA) particles (Dantec Dynamics). These particles contain rhodamine and are used for the PIV experiment. Their mean diameter is approximately $10 \mu \mathrm{m}$ and the impedance ratio between water and PMMA is $\frac{Z_{w}}{Z_{\mathrm{P} M \mathrm{AM}}} \approx \frac{1}{2}$.

- Polydisperse silica particles (Arena) with a wide diameter distribution centred at $67.5 \mu \mathrm{m}$. The impedance ratio is $\frac{Z_{w}}{Z_{S}} \approx \frac{1}{10}$.

- Monodisperse silica particles with a narrow diameter distribution centred at $56.5 \mu \mathrm{m}$ are obtained by sifting the polydisperse silica particles.

Figure 3 shows the diameter distributions of these three particle populations.

\section{Experimental procedures}

This section presents the recording system and then the signal processing to optimize the USV experiments.

\subsection{The device}

The ultrasonic transducer frequency is chosen according to the diameter distribution of the ultrasound scatterers. Actually, the Rayleigh scattering theory clearly indicates that a speckle is observed for $d \ll \lambda$, where $d$ is the mean diameter of the particles [12]. 2.25 and $25 \mathrm{MHz}$ transducers are thus used to study the sedimentation of the silica and PMMA particles. The wavelengths are $\lambda=670 \mu \mathrm{m}$ and $\lambda=60 \mu \mathrm{m}$, respectively. The pulser-receiver generator must be adjustable to process the speckle signal.

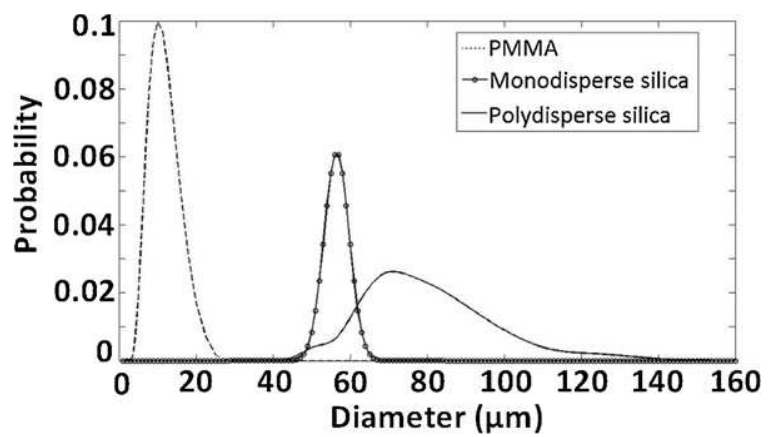

Figure 3. Diameter distributions of the PMMA, the polydisperse silica and the monodisperse silica particles.

Its main parameters are the bandwidth BW coupled with the attenuation $A$ and the gain $G$ which limit the transducer frequency range, the damping current $C$ controlling the shape of the ultrasonic pulse, and finally, the transducer energy $E$. For the following results, the setting is: $A=-1 \mathrm{~dB}, G=$ $54 \mathrm{~dB}, C=50 \Omega$ and $E=32 \mu \mathrm{J}$ for both the transducers. The bandwidths were fixed at $1 \leqslant \mathrm{BW} \leqslant 10 \mathrm{MHz}$ and $10 \leqslant \mathrm{BW} \leqslant 50 \mathrm{MHz}$ for the 2.25 and $25 \mathrm{MHz}$ transducers, respectively, and are determined using the Shannon theorem [14]. Previous experiments or theory are necessary to evaluate the particles velocity in order to adjust the PRF. In our case, the spherical particles velocity is estimated considering Stockian flows:

$$
v(d)=\frac{\rho g d^{2}}{18 \eta}\left(1-\frac{\rho_{w}}{\rho}\right),
$$

where $\rho$ and $\rho_{w}$ are the volume weight of the particles and water, and $\eta$ is the water viscosity. Consequently, the PRF was fixed at $200 \mathrm{~Hz}$ for the PMMA particles and $1 \mathrm{kHz}$ for the silica particles.

The signal recording is carried out with the oscilloscope using sampling frequencies of 500 and $50 \mathrm{MHz}$ for the PMMA and silica particles, respectively.

\subsection{Signal processing}

To accurately measure the velocity in terms of the sedimentation time, ten $S(\vec{r})$ are successively recorded 200 times for each experimental condition. Between two acoustical pulses, the whole $S(\vec{r})$ is divided into $N$ windows where each window has a percentage of overlap with the previous windows varying between $50 \%$ and $100 \%$. The $N$ measurement positions of the velocity define the space resolution of USV. For an $80 \%$ overlap, $N$ is given by

$$
N=\sum_{i} N_{i} \approx \frac{5 L}{n \lambda}+1,
$$

where $L$ is the cell height. Typically, $N=625$ for PMMA with $n=4$. The cross-correlation function is then computed automatically with the Matlab ${ }^{\circledR}$ program for each speckle window couple $\left\{N_{i}\left(k t_{\mathrm{PRF}}\right) ; N_{i}\left((k+1) t_{\mathrm{PRF}}\right)\right\}$, where $k$ is the number of acoustic pulses, $k \in[1,9]$.

Figure 4 shows two 3D representations of $S(\vec{r})$ as a function of $k$ and the flowing time $t$. The first representation 

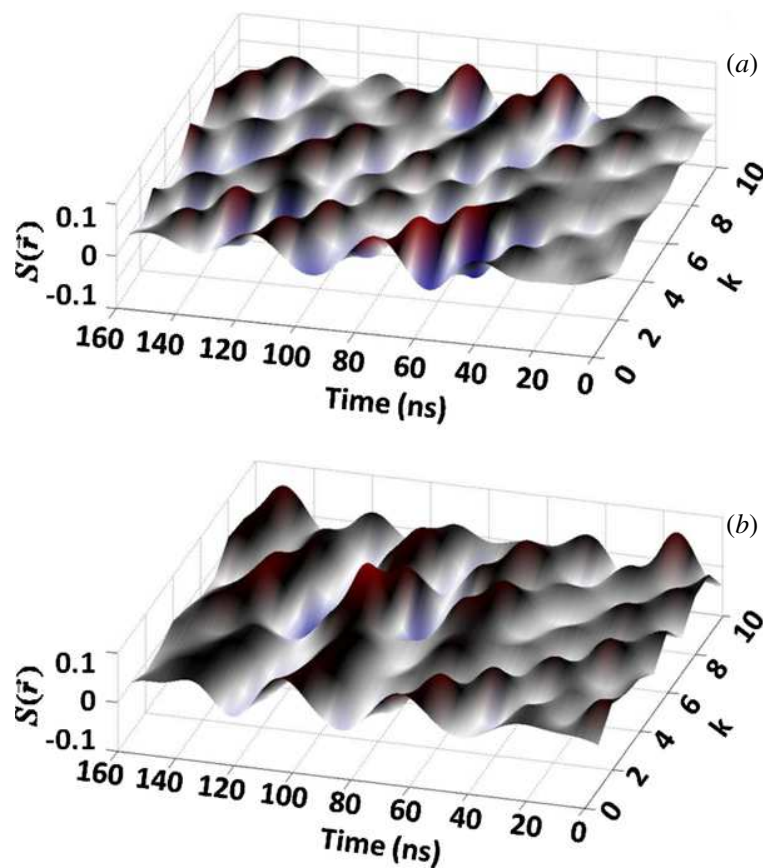

Figure 4. 3D representations of the speckle signal $S(\vec{r})$ as a function of time and the number of acoustic pulses. (a) Example of a coherent speckle. (b) Example of a noisy speckle.

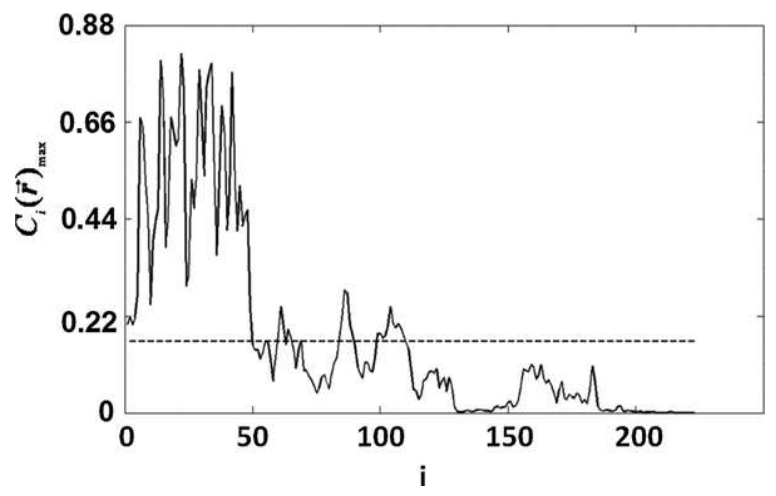

Figure 5. Maximum of the mean cross-correlation $C_{i}(\vec{r})_{\max }$ as a function of the number of speckle windows $i$. The dashed line represents the criteria of $20 \%$ of the maximum of $C_{i}(\vec{r})_{\max }$.

(figure 4(a)) presents a high level of correlation for which the $C(\vec{r})$ give a well-defined maximum. The second (figure $4(b)$ ) representation presents a low level of coherence and the $C(\vec{r})$ are characterized by secondary maxima with amplitude close to the main peak. The latter speckle image is predominant in USV and requires specific procedures to be analyzed: for each $i$ speckle windows, we calculated the averaged cross-correlation functions $C_{i}(\vec{r})$, determined the maximum $C_{i}(\vec{r})_{\max }$ and plotted the maxima according to $i$ (figure 5). At this stage, we defined two criteria to discard cross-correlations with a small intensity, and this results from data with a poor signal-to-noise ratio. Indeed, destructive interference or absence of scatters may lead to signal levels too small to be analyzed (figure 6). Then, a first criterion allows leaving

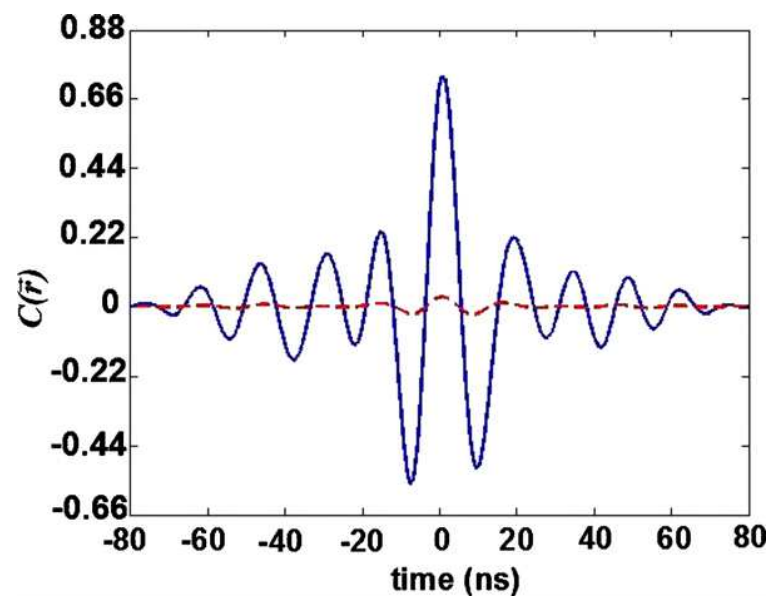

Figure 6. Examples of two cross-correlations: the first one has an acceptable intensity to be analyzed and the second has too small an intensity and is to be discarded using a criterion that leaves out all the cross-correlations given a $C_{i}(\vec{r})_{\max }$ value less than $20 \%$ of the curve of the maxima (see figure 5).

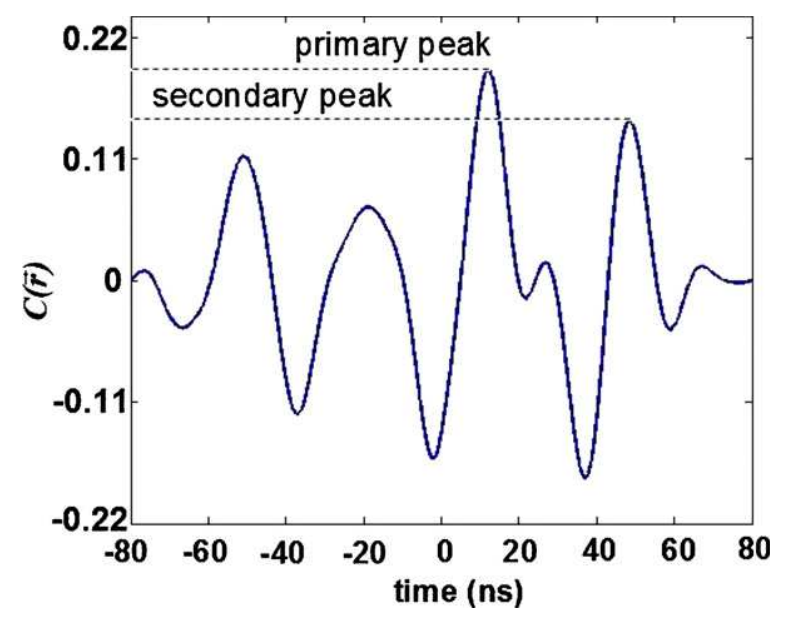

Figure 7. Example of a cross-correlation obtained from the speckles measured with a low signal-to-noise ratio. In the data analysis, we used a criterion to leave out all the cross-correlations having a secondary-primary peak amplitude ratio greater than $50 \%$.

out all the cross-correlations given a $C_{i}(\vec{r})_{\max }$ value of less than $20 \%$ of the curve of the maxima. Furthermore, we had to eliminate all the cross-correlations obtained from the speckle measured with a low signal-to-noise ratio (figure 7). A second criterion was determined experimentally. It allows leaving out all the cross-correlations having a secondary-primary peak amplitude ratio greater than $50 \%$. From all $C_{i}(\vec{r})$ verifying both the criteria, the velocities were finally calculated.

The space resolution is governed by the length $\Delta r=n \lambda$ and the percentage of overlapping of the speckle windows used for cross-correlations. In this paper, we varied the percentage of overlapping between $50 \%$ and $100 \%$ and studied the effect of the length of the speckle windows for $n=4,8$ and 16 for PMMA and $n=4$ and 8 for silica particles, yielding $240 \leqslant \Delta r \leqslant 960 \mu \mathrm{m}$ for the PMMA and $2.6 \leqslant \Delta r \leqslant$ 

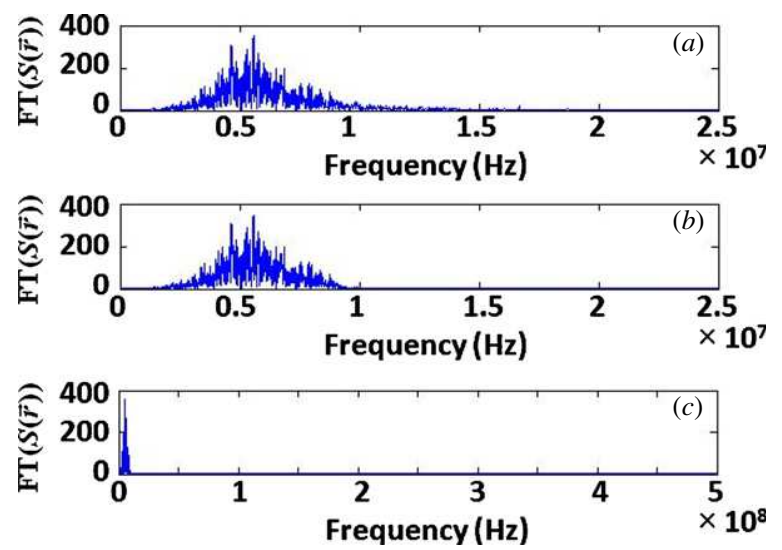

Figure 8. Fast Fourier transform of the speckle signal for silica particles: $(a)$ without offset; $(b)$ with a numerical filter between 1 and $10 \mathrm{MHz} ;(c)$ with a numerical filter between 1 and $10 \mathrm{MHz}$ and zero padding processing. The new sampling frequency is $10 \mathrm{GHz}$.

$5.2 \mathrm{~mm}$ for the silica particles. The results will be discussed in the next section.

If the measured minimum velocity is obtained from the minimum of $\delta t$, the maximum measured velocity is estimated from the width of the speckle windows:

$$
v_{\max }=\frac{n \lambda}{2 t_{\mathrm{PRF}}} \text {. }
$$

Then, the USV technique presents very important dynamics, authorizing velocity measurements along the acoustic axis in the range $\left[1.5 \times 10^{-5}-4.8\right] \mathrm{m} \mathrm{s}^{-1}$ for the PMMA and $[1.5 \times$ $\left.10^{-5}-5.3\right] \mathrm{m} \mathrm{s}^{-1}$ for the silica particles.

In terms of signal processing, we established a three-stage procedure. First, the offset signal has to be removed. Second, a numerical filter is applied with the Matlab ${ }^{\circledR}$ software between 10 and $50 \mathrm{MHz}$ with $-60 \mathrm{~dB}$ of attenuation in order to suppress the non-speckle signal. Subsequently, to obtain a temporal resolution of $0.1 \mathrm{~ns}$ with the 50 and $500 \mathrm{MHz}$ sampling frequencies, the speckle data are re-sampled with zero padding signal processing [15]. This consists of extending a measured spectrum with zero in order to reach a wide sampling frequency and obtain a better time resolution. In this paper, the sampling frequency increases from $50 \mathrm{MHz}$ to $10 \mathrm{GHz}$ for the silicate particles and from $500 \mathrm{MHz}$ to $10 \mathrm{GHz}$ for the PMMA particles. Figure 8 shows the numerical filter and the zero padding effects on a speckle signal Fourier transform. The three stages are necessary to obtain a speckle signal ready for analysis.

\section{Results and discussion}

Figure 9 compares the experimental velocity distributions of the three particle families for $n=4$ and for $50 \%$ overlap of the speckle windows with those calculated theoretically. The mean velocities and standard deviations are assembled in table 1 . One can observe that the three velocity distributions follow a Gaussian law in an initial approximation and the mean velocities for the PMMA particles, the polydisperse and the
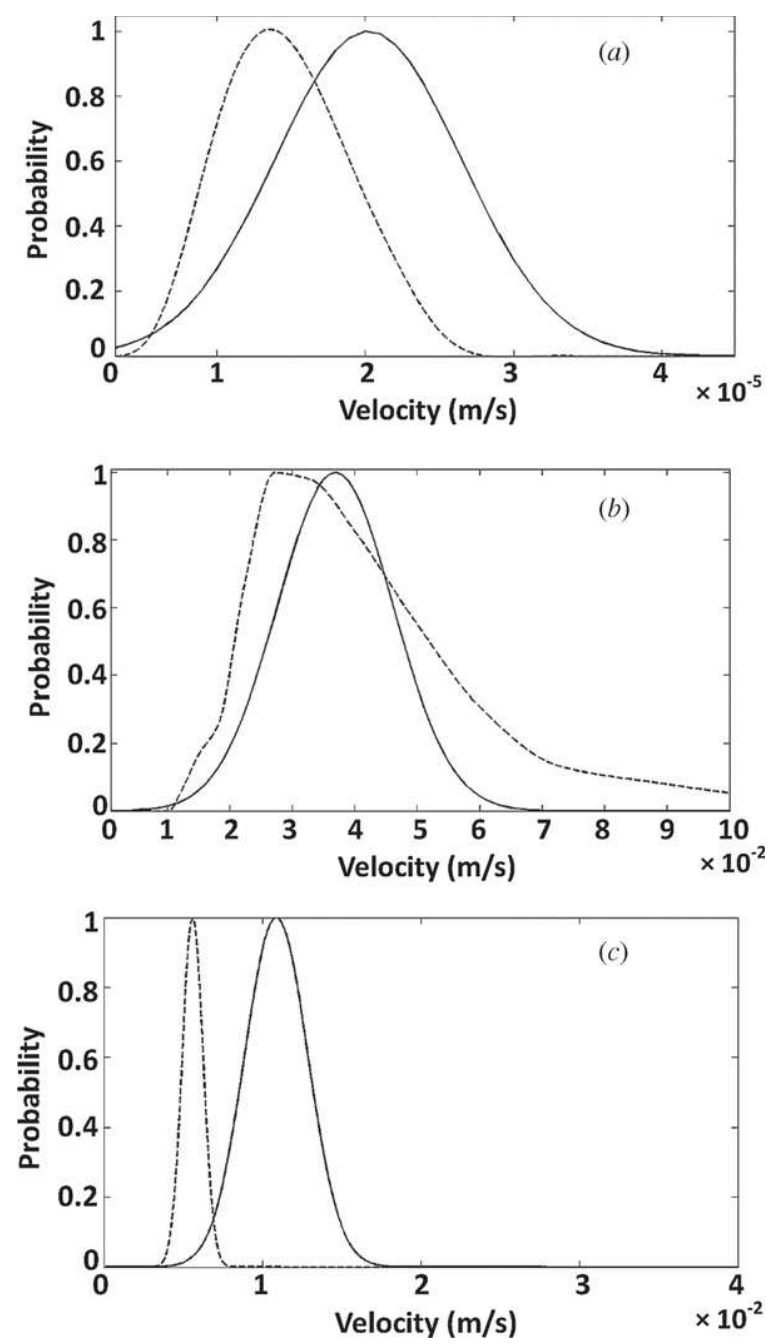

Figure 9. Velocity distribution of $(a)$ the PMMA, $(b)$ the polydisperse silica and $(c)$ the monodisperse silica particles for a length of the speckle window corresponding to $n=4$ and $50 \%$ overlap. The dashed lines represent the theoretical Stockian velocity and the solid lines represent the experimental values.

monodisperse silica particles are $\bar{v}_{\text {PMMA }}=20 \pm 6 \mu \mathrm{m} \mathrm{s}^{-1}$, $\bar{v}_{\text {silica }}=3.69 \pm 0.92 \mathrm{~cm} \mathrm{~s}^{-1}$ and $\bar{v}_{\text {silica }}^{\text {mono }}=1.12 \pm 0.29 \mathrm{~cm} \mathrm{~s}^{-1}$. These results allow checking that USV has significant dynamics and is able to measure the velocity over six decades. From the size distributions, it is possible to analyze the theoretical velocity distribution in the Stockian regime (figure 9). Comparing both theoretical and experimental distributions, USV experiments clearly give mean velocities shifted towards higher values. This shift is emphasized for the polydisperse silica particles and can be explained by considering that the multiple ultrasound scattering efficiency is obtained for the larger diameter particles while the theoretical velocity calculus gives the same weight for all the particles. The USV results in figure 9 also show the effect of the size distribution of the silica particles. The mean velocity $(1.12$ and $3.69 \mathrm{~cm} \mathrm{~s}^{-1}$ for the monodisperse and polydisperse silica particles) depends on the mean particle diameter (56.5 and 
Table 1. Values of the mean velocities and the standard deviations for the PMMA, the polydisperse silica and the monodisperse silica particles for different lengths of the speckle windows. The overlap of the speckle windows was fixed at $50 \%$.

\begin{tabular}{lrrlllll}
\hline Particles & \multicolumn{3}{c}{ Mean velocity $\left(\mathrm{m} \mathrm{s}^{-1}\right)$} & & \multicolumn{3}{c}{ Standard deviation $\left(\mathrm{m} \mathrm{s}^{-1}\right)$} \\
\cline { 2 - 3 } & $n=4$ & $n=8$ & $n=16$ & & $n=4$ & $n=8$ & $n=16$ \\
\hline Polydisperse silica & $3.69 \times 10^{-2}$ & $3.50 \times 10^{-2}$ & - & & $0.92 \times 10^{-2}$ & $1.08 \times 10^{-2}$ & - \\
Monodisperse silica & $1.12 \times 10^{-2}$ & $1.21 \times 10^{-2}$ & - & & $0.29 \times 10^{-2}$ & $0.40 \times 10^{-2}$ & - \\
PMMA & $2.0 \times 10^{-5}$ & $1.8 \times 10^{-5}$ & $1.8 \times 10^{-5}$ & & $0.6 \times 10^{-5}$ & $0.9 \times 10^{-5}$ & $0.7 \times 10^{-5}$ \\
\hline
\end{tabular}
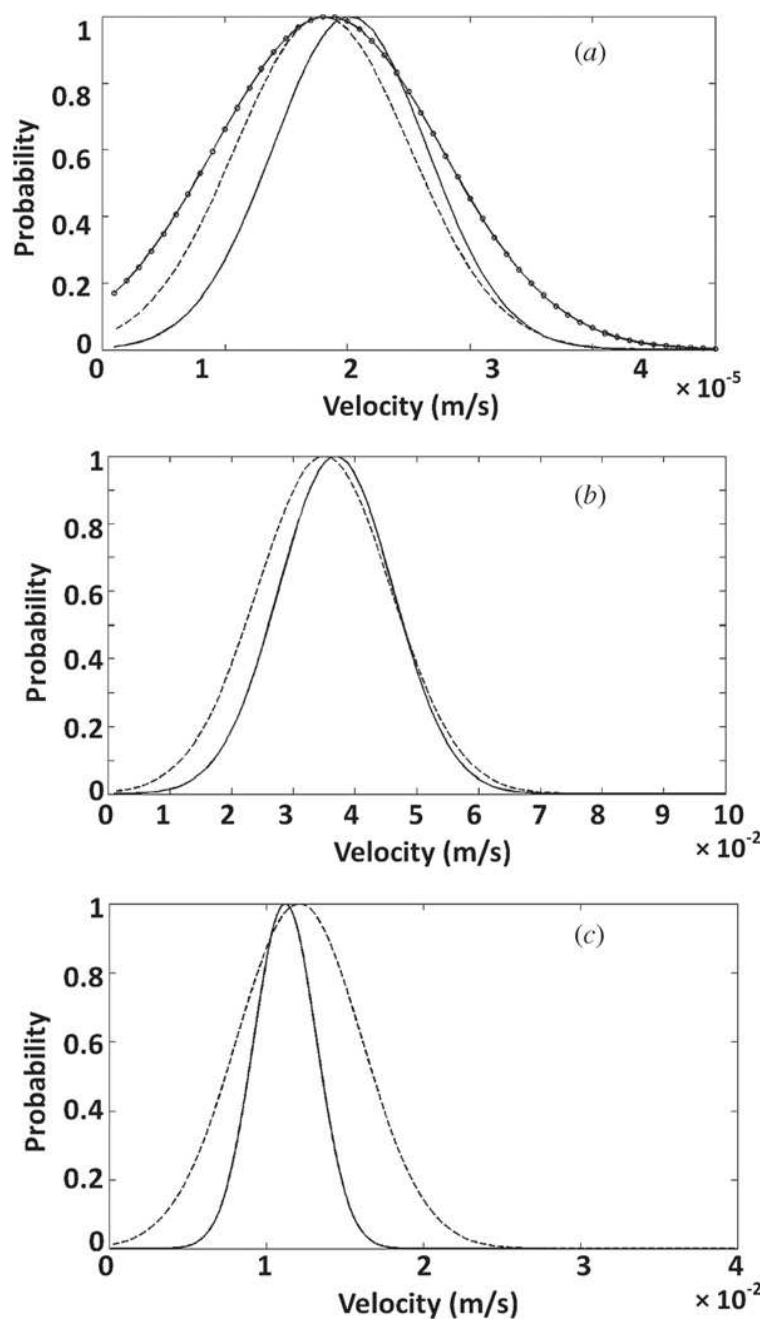

Figure 10. Velocity distributions of ( $a$ ) the PMMA particles for three lengths of the speckle window: $n=4$ (solid line), 8 (dotted line) and 16 (dashed line); $(b)$ the polydisperse silica particles for two lengths of the speckle window: $n=4$ (solid line) and 8 (dashed line); (c) the monodisperse silica particles for two lengths of the speckle window: $n=4$ (solid line) and 8 (dashed line). The overlap of the speckle windows was fixed at $50 \%$.

$67.5 \mu \mathrm{m}$, respectively), while the velocity standard deviation increases with the particle size distribution.

We compared the USV results with the PIV results for the PMMA particles. In a similar case of sedimentation in water, Roudet measured a velocity of approximately $30 \mu \mathrm{m} \mathrm{s}^{-1}$ by PIV [16], a value in agreement with that measured by USV. The difference between the two values

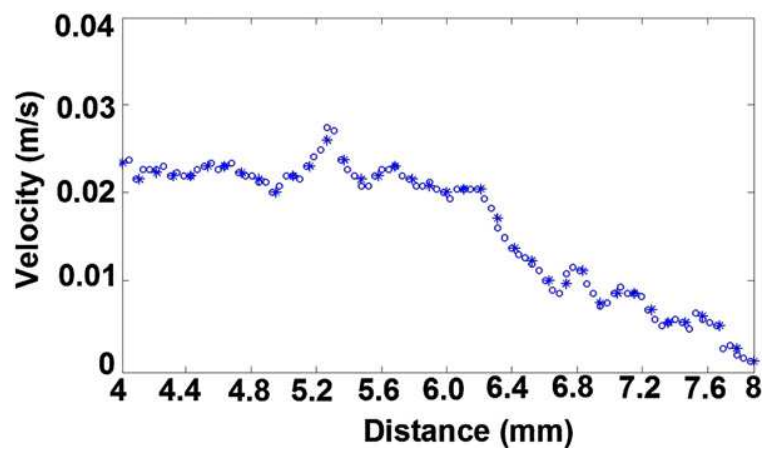

Figure 11. Velocity profile along the acoustical axis for polydisperse-silica-particles-measured cross-correlation of the $80 \%$ overlap speckle windows (circles) and the 50\% overlap speckle windows (stars).

can be understood by considering that the experiments were implemented with two different seeding procedures. Indeed, in the experiments of Roudet, the PMMA particles were dispersed in water beforehand to constitute a master batch, which was later diluted for each series of experiments. This procedure promotes cluster formation in the master batch which would sediment with a higher velocity. We found that using a funnel to disperse the particles leads to reproducible results, thus avoiding cluster formation.

The USV space resolution is studied by systematically changing the percentage of overlap between $50 \%$ and $100 \%$ and the speckle window length $\Delta r$ (for $n=4,8$ and 16) for the three particle families. Figure 10 shows that at $50 \%$ overlap, the mean velocity is less affected by a change of $\Delta r$. On the other hand, the velocity distribution width changes with the speckle window length and the standard deviation seems to increase with $n$. This effect is due to a widening of the characterized area in the sedimentation cell. Moreover, for a large size distribution, particle segregation could occur during the sedimentation process, yielding a broadening of the velocity distribution.

At the same time, we found that the value of the mean velocity is not affected by the percentage of overlap but the standard deviation increases drastically when the percentage is greater than $80 \%$. If the best space resolution is given by the value of the particle displacement during the time $t_{\mathrm{PRF}}$ (approximately $15 \mu \mathrm{m}, 27 \mu \mathrm{m}$ and $75 \mathrm{~nm}$ for the monodisperse silica particles, the polydisperse silica particles and the PMMA particles, respectively) corresponding to $99 \%$ overlap, this result could be understood according to the limitations of the software used to overlap the speckle windows. As an example, figure 11 compares the velocity sedimentation profile 
for the polydisperse silica particles at $50 \%$ and $80 \%$ overlap and $n=4$. We observe that the velocity decreases with the distance, a result that can be explained by the particle diameter effects on the sedimentation velocity. As expected, the data at $50 \%$ overlap coincide with those at $80 \%$. However, the space resolution increases with the percentage of overlap. Typically, the space resolution is approximately 1340 and $536 \mu \mathrm{m}$ for the silica particles and approximately 120 and $48 \mu \mathrm{m}$ for the PMMA particles at $50 \%$ and $80 \%$ overlap.

\section{Conclusion}

On the basis of the particle sedimentation experiments, we have improved USV with the aim of presenting the advantages and limitations of this technique. We have shown that three numerical stages are necessary before data processing: offset subtraction, numerical filtering and re-sampling by the zero padding method. Moreover, two cross-correlation criteria were defined to optimize data processing. We studied the effects of the speckle window length and the percentage of overlapping of the speckle windows on the measured velocities and found that the results are more accurate with the smallest window length and a percentage of overlapping varying between $50 \%$ and $80 \%$.

In order to optimize USV, we measured sedimentation velocities of three different particle families: PMMA, monodisperse and polydisperse silicate particles, which are characterized by different density, acoustical impedance and size distribution. For the USV experiments, 2.25 and $25 \mathrm{MHz}$ transducers were used. In our signal processing conditions, we reached a temporal resolution of $0.1 \mathrm{~ns}$ for both the transducers. The best space resolutions, functions of the acoustical wavelength, were obtained for $n=4$ and for $80 \%$ overlap. We found a space resolution of $48 \mu \mathrm{m}$ for the PMMA particles and $536 \mu \mathrm{m}$ for the silica particles. We measured velocities varying between $20 \mu \mathrm{m} \mathrm{s}^{-1}$ and $3 \mathrm{~cm} \mathrm{~s}^{-1}$ for the three particle families, showing the wide dynamic potentiality of USV. In addition, we verified the coherence of results between the USV and PIV experiments and between the USV experiments and theory. In conclusion, following the different steps of signal processing and data analysis described in this paper, we have shown that USV is a useful method for the study of particle sedimentation and velocity profile in full flow.

\section{Acknowledgments}

The authors would like to thank V Roig, F Risso and M Roudet for numerous fruitful discussions. They would also like to thank C André for the characterization of monodisperse silica particles.

\section{References}

[1] Cummins H Z and Pike E R 1977 Photon Correlation Spectroscopy and Velocimetry (New York: Plenum)

[2] Berne B J and Pecora R 2000 Dynamic Light Scattering (Toronto, Canada: General Publishing)

[3] Gens F, Remenieras J P, Patat F, Diridollou S and Berson M 2000 Potential of a high-frequency correlation method to study skin blood flow Skin Res. Technol. 6 21-6

[4] Bonnefous O 2001 Blood flow and tissue motion with ultrasound for vascular applications C. R. Acad. Sci., Paris 2 1161-78

[5] Atkinson P and Woodcock J P 1982 Doppler Ultrasound and Its Use in Clinical Measurement (London: Academic)

[6] Kim H B, Hertzberg J R and Shandas R 2004 Development and validation of echo PIV Exp. Fluids 36 455-62

[7] Nakajima M, Itoh T, Shingyouuchi M, Akiyama I and Yuta S 1988 Ultrasonic speckle velocimetry Proc. IEEE Ultrason. Symp. 2 1007-12

[8] Manneville S, Sandrin L and Fink M 2001 Investigating a stretched vortex with ultrafast two-dimensional ultrasonic speckle velocimetry Phys. Fluids 13 1683-90

[9] Gharib M and Beizaie M 2003 Correlation between negative near-wall shear stress in human aorta and various stages of congestive heart failure Ann. Biomed. Eng. 31 678-85

[10] Manneville S, Bécu L and Colin A 2004 High-frequency ultrasonic speckle velocimetry in sheared complex fluids Eur. Phys. J. Appl. Phys. 28 361-73

[11] Cowan M L, Jones I P, Page J H and Weitz A 2002 Diffusing acoustic wave spectroscopy Phys. Rev. E 65066605

[12] Born M and Wolf E 1964 Principles of Optics (Cambridge: Cambridge University Press)

[13] Fink M, Mallart R and Cancre F 1990 The random phase transducer: a new technique for incoherent processing-basic principles and theory IEEE Trans. Ultrason. Ferroelectrics Freq. Control 37 54-69

[14] Proakis J G and Manolakis D G 1996 Advanced Digital Signal Processing International edn (New York: McGraw-Hill)

[15] Carlson A B 1986 Communication Systems (New York: Macmillan)

[16] Roudet M 2008 Hydrodynamique et transfert de masse autour d'une bulle confinée entre deux plaques $P h D$ Thesis University of Toulouse, France http://ethesis.inp-toulouse.fr/archive/00000806/ 